the pediatric departments. For x-ray sessions in $2017(23,448$ in working hours and 1823 business cases), most of the x-ray sessions were done on Tuesday (28\%), with fewest done on Thursday (10\%). Upon analyzing the data, the calculated average patient waiting time was 22 minutes. Further analysis showed that $82 \%$ of patients had met the waiting time threshold, which is 30 minutes. The medical imaging unit located in the ACC building contains five x-ray rooms; $41 \%$ of all sessions in 2017 had been performed in room number 3 .

Conclusion Based on the analysis, the team determined that the process is stable and predictable. However, there are several areas for improvement that we would recommend focusing on:

1. The distribution of the clinic during the week, Thursdays have the fewest clinics; therefore, it is the least utilized day.

2. The distribution of the rooms to perform X-ray sessions; room number 3 is used most out the five rooms (41\%) and room 1 is used the least $(11.76 \%)$.

3. Waste of requesting X-ray tests overall hospital. The team suggest establishing small projects to highlight and improve the previous areas and review current staffing and rostering allocations.

\section{DEVELOPING A SPECIALTY-SPECIFIC HANDOFF TOOL: A NATIONAL ELECTRONIC DELPHI STUDY}

Khaled Alrajhi, Abdulmohsen Alsaawi. Emergency Medicine, Ministry of National Guard Health Affairs, Riyadh, Saudi Arabia

10.1136/bmjoq-2019-PSF.77

Background Handoffs at the end of clinical shifts are a daily routine in emergency department settings and are considered by most authorities as a common source of risk and potential harm to patients. There is a need to standardize the patient handoff process to reduce related human errors. This study aimed to use an electronic Delphi method to identify the core elements essential for an emergency department physician-tophysician handoff.

Methods Panelists were required to be board-certified emergency physicians with no less than 3 years of post-board experience. An electronic Delphi-style study was performed over four rounds. The first was to identify elements and categorize them into domains, and the remaining three to score and debate individual elements. Items were anonymously scored on how frequently each element was required during handoffs, from 1 'rarely required' to 10 'always required'. Panelists were able to add and respond to arguments as well.

Results 29 emergency physicians were enrolled in the panel and all panelists completed the entire Delphi process. The top five rated handoff elements were the chief complaint history, patient identification, clinical stability, working diagnosis, and consulting services involved. Panel scores showed less variability as rounds progressed and the final list of elements had a high reliability score (Cronbach's alpha 0.93).

Conclusion The study methods yielded an itemized and ranked list of elements that is easy to implement as a checklist or in forms and could be used to standardize patient handoffs by emergency physicians. Arbitrary cutoff values may be used to design a handoff tool based on the results of such studies. These cutoffs could help decide which elements to include or which elements may be mandatory in a proposed handoff tool. These methods may be adapted to develop standardized handoff frameworks that serve other specific disciplines or practice settings. 